\title{
Research on Affective Computing Model in E-Learning System
}

\author{
Ruiming Zhao ${ }^{1}$, Zhenhui Ren ${ }^{1}$, Li Zhang ${ }^{1}$, and Ruiguo Qin ${ }^{2}$ \\ ${ }^{1}$ Mechanical and Electrical College, Hebei Agriculture University, Baoding \\ 071001, China \\ ${ }^{2}$ Hebei Baoding TV, Baoding 071050, China \\ powerzrm@yahoo.com.cn
}

\begin{abstract}
Emotion deficiency was a hot topic research in current E-learning system. A lot of negative effects were analyzed and corresponding countermeasures were proposed in the paper. Basing on it, affective computing model was set up and used in the traditional E-learning system. Affective computing model was constructed by using audiovisual emotion, which took both facial and audio features as input data. Rough set theory was used in audiovisual emotion recognition. Our simulation experiment results showed that Rough set theory was effective in emotion recognition, and a high recognition rate was resulted.
\end{abstract}

Keywords: Affective Computing, Emotion recognition, Rough Set, Feature Selection.

\section{Introduction}

With the development and widespread of network, E-learning has become one of most important ways of educating and researching. E-learning can break through the limit of space and time, reduce learning cost and improve learning efficiency [1]. People pay more and more attention to it.

Although the current E-learning systems have many merits, the phenomenon of emotion deficiency in the current E-learning system is existed. How to measure cognitive emotion of learners in the E-learning system and realize harmonious emotion interaction becomes an important research topic in the distance education [2]. Aiming at the problem of emotion deficiency in E-learning, domestic and abroad scholars bring forward some strategies as follows:

(1) Designing the emotional network curriculums.

(2) Implementing exploring and cooperative learning.

(3) Implementing blended learning.

(4) Improving learning supporting service system.

The application of above strategies have avoided emotion deficiency in certain degree, but learner's emotion state cannot be tracked accurately, the corresponding emotional encouragement and compensation also cannot be provided according to specific emotion state, which cannot help the learner to solve emotion deficiency fundamentally. 
Affective computing is a hot topic in Artificial intelligence, it is computing that related to, arise from, or deliberately influence emotion [3], which is firstly proposed by Professor Picard at MIT in 1997.Affective computing consists of recognition, expression, modeling, communicating and responding to emotion [4].in this components, emotion recognition is one of the most fundamental and important modules. It is always based on facial and audio information.

Basing on it, affective computing model was set up and used in the traditional Elearning system. Both facial expression recognition and speech emotion recognition are used to construct affective computing model. Rough set theory was used in audiovisual emotion recognition.

\section{Affective Computing Model}

The affective computing model is Fig.1. The system model is composed of several modules as follows:

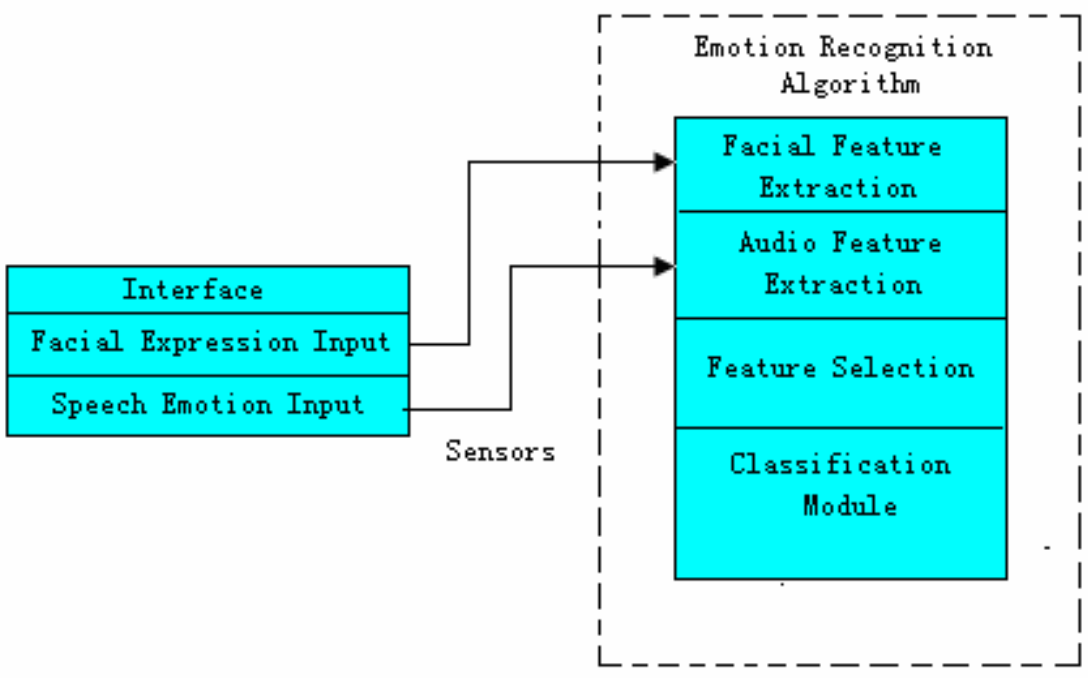

Fig. 1. Affective Computing Model

Interface module: affective computing input will be added to human machine interface of traditional E-learning system (both facial expression recognition input and emotion recognition of speech input), which collects learners' emotion feedback information primarily, thus emotion compensation is realized.

Emotion Recognition algorithm module: emotion recognition algorithm module is composed of input, pre-processing, feature extraction, feature selection, classification and output. Firstly, facial image and audio information of human are taken as input data through some relevant sensors. Then, audio feature and facial feature are extracted from input data. Furthermore, valuable features from all features are 
selected for emotion recognition. Finally, the overall emotion is recognized by using emotion recognition algorithm based on Rough Set theory.

\section{Emotion Recognition Algorithm}

\subsection{Audio Feature Extraction}

The 37 secondary (statistical) speech features listed in table 3 are often used in speech emotion recognition systems, and the 37 features are taken in our emotion recognition module [5].

\subsection{Facial Feature Extraction}

In this paper, AAM is adopted to locate feature points. On the other hand, the MPEG4 standard is a popular standard for feature point selection. It extends FAGS to derive Facial Definition Parameters (FDP) and Facial Animation Parameters (FAP). FAP has been used widely in facial animation for its good performance on compression in recent years. Besides, the FDP and low level FAP constitute a concise representation of a face. They are adequate for basic emotion recognition because of the varieties of expressive parameter. In FAP, 66 low level parameters are defined to describe the motion of a human face. Among 66 parameters, 52 parameters are chosen to represent emotion in our recognition system as shown in Fig.2, because some parameters have not much impact on emotion. Thus, a feature point set including 52 feature points is defined in the image sequence. Based on the feature points, feature distance can be calculated as the features for emotion recognition.

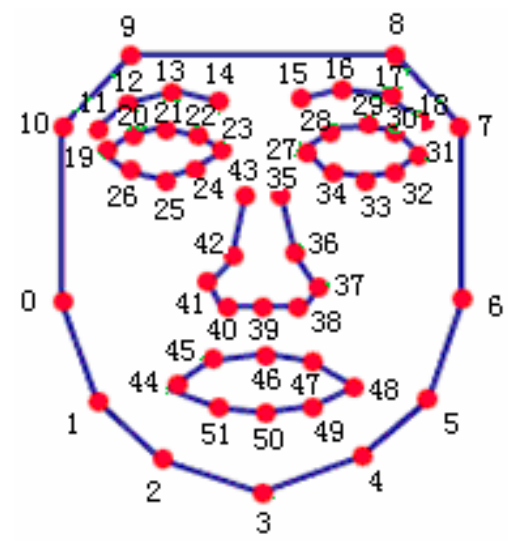

Fig. 2. Feature points

\subsection{Feature Selection}

Feature selection is the key part of emotion algorithm recognition module. The purpose of feature selection is to select valuable features for emotion recognition from 
all features. In this module, features for emotion recognition are decreased. Thus, the complexity and cost for the following classification procedure are reduced. It would improve the efficiency of the whole system.

In our emotion algorithm recognition module, feature selection is based on the rough set theory. The attribute reduction algorithm adopted to select features is introduced in RSFSA, and results of experiment shows the algorithm is effective.

\subsection{Classification Module}

The classification techniques used in existing emotion recognition algorithm module include template-based classification, rule-based classification, ANN-based classification, HMM-based classification, Bayesian classification, SVM-based classification, etc.

In our emotion recognition algorithm module, classification rules created according to rough set reduction algorithm are taken as the classifier.

\section{Conclusion}

In this paper, affective computing model was set up and used in the traditional Elearning system. Both facial expression recognition and speech emotion recognition are used to construct affective computing model. Rough set theory was used in audiovisual emotion recognition. Depending on these features, an average recognition rate of $79.2 \%$ is achieved. In the future, based on the selected features, other effective classification method will be used. And multi-module emotion recognition including facial, audio, and other features such as gesture will be studied [6].

\section{References}

1. Luo Qi.: Research on Application of Association Rule Mining Algorithm in Learning Community. Proceedings of CAAI-11, Wuhan, 2005, 1458-1462.

2. Ma Xirong.: Research on harmonious man-machine interaction model. Computer science, 2005.

3. R.W.Picard.: Affective Computing. Cambridge. MIT Press, 1997.

4. R.W.Picard.: Affective Computing, Challenges. Cambridge. International Journal of Human Computer studies, 59(1), 2003, 55-64.

5. D.Ververidis, et al.: Automatic emotion speech classification. Proceedings of the ICASSP2004, 2004, 593-596.

6. Q.Cai, A.Mitche and J.K.Aggarwal.:Track Human Motion in an Indoor Environment. Proceedings of 2nd international conference Image Processing, 2003. 